(C) 2017 by the Arizona Board of Regents on behalf of the University of Arizona. This is an Open Access article, distributed under the terms of the Creative Commons Attribution licence (http://creativecommons. org/licenses/by/4.0/), which permits unrestricted re-use, distribution, and reproduction in any medium, provided the original work is properly cited.

\title{
USING STABLE ISOTOPES AND A BAYESIAN MIXING MODEL (FRUITS) TO INVESTIGATE DIET AT THE EARLY NEOLITHIC SITE OF CARDING MILL BAY, SCOTLAND
}

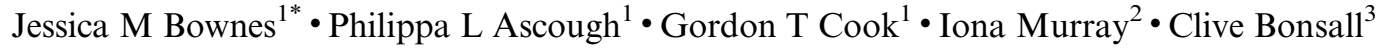 \\ ${ }^{1}$ Scottish Universities Environmental Research Centre, Rankine Avenue, Scottish Enterprise Technology Park, \\ East Kilbride G75 0QF, United Kingdom. \\ ${ }^{2}$ Historic Environment Scotland, Longmore House, Salisbury Place, Edinburgh EH9 1SH, United Kingdom. \\ ${ }^{3}$ Archaeology, University of Edinburgh, Old Medical School, 4 Teviot Place, Edinburgh EH8 9AG, United Kingdom.
}

\begin{abstract}
We present $\delta^{13} \mathrm{C}, \delta^{15} \mathrm{~N}$, and $\delta^{34} \mathrm{~S}$ measurements on archaeological human and animal bone collagen samples from a shell midden dating to the Neolithic ca. 4000-3500 cal BC, together with measurements on modern fish and shellfish. These data were used in conjunction with the Bayesian mixing model, Food Reconstruction Using Isotopic Transferred Signals (FRUITS), to reconstruct human diet at the site. We demonstrate the importance of using a geographically appropriate faunal baseline in stable isotope paleodietary studies, and suggest that Neolithic individuals at this site consumed up to ca. $21 \%$ of dietary protein from marine resources, despite stable isotope ratios that imply a wholly terrestrial diet. This marine resource consumption does not significantly shift the radiocarbon $\left({ }^{14} \mathrm{C}\right)$ dates of these individuals, so although we must consider the use of marine resources at the site, the chronology that has previously been established is secure. The $\delta^{13} \mathrm{C}$ and $\delta^{15} \mathrm{~N}$ measurements from the archaeological herbivore bone collagen indicate that it is unlikely they ate plants enriched with fertilisers such as manure or seaweed. The $\delta^{34} \mathrm{~S}$ values reveal a sea-spray effect; therefore, in this instance, $\delta^{34} \mathrm{~S}$ cannot be used as a dietary indicator but can be used to demonstrate the likely locality of the fauna.
\end{abstract}

KEYWORDS: stable isotopes, paleodiet, Mesolithic, Neolithic, Scotland.

\section{INTRODUCTION}

The Mesolithic-Neolithic transition marked a profound shift in socioeconomic patterns, which is represented in the archaeological record by stark changes in material culture and subsistence practices. The Neolithic brought a fundamental change in human lifestyle, reflected in the introduction of farming, new technology including pottery and ground stone artifacts, and distinctive forms of architecture and burial practice.

The nature and timing of the Mesolithic-Neolithic transition remains a much-debated topic in archaeology. Recent aDNA studies have shown that early farmers in central and southwestern Europe show a genetic affinity to populations in the Near East, with the implication that demographic expansion and migration played an important role in the appearance of the Neolithic in these regions, although an admixture with local foragers is also indicated (e.g. Haak et al. 2010; Olalde et al. 2015). Palaeogenetic research into the transition in the British Isles is less advanced and archaeological opinion is still divided over whether colonisation from mainland Europe (Sheridan 2010) or indigenous adoption (Thomas 2008) was the main driver of Neolithization.

Regardless of the eventual outcome of the debate over the nature and timing of the transition in the British Isles, it is pertinent that we seek answers to more detailed questions about human lifestyle at this key juncture in prehistory. The extent of dietary change associated with the Mesolithic-Neolithic transition in Scotland is one such question that remains controversial. Stable isotope analysis for dietary reconstruction has been employed to investigate these changes (Richards and Mellars 1998), but is constrained by the scarcity of Mesolithic human remains from Scotland. Evidence of a chronological overlap in Mesolithic and Neolithic

\footnotetext{
*Corresponding author. Email: j.bownes.1@research.gla.ac.uk.
} 
lifestyles comes from the shell midden of Cnoc Coig, Oronsay, which is usually attributed to the Late Mesolithic. Charlton et al. (2016) used ZooMS, stable isotope analysis and radiocarbon $\left({ }^{14} \mathrm{C}\right)$ dating to identify two human bones from the midden with stable carbon and nitrogen isotope values that reflect the consumption of a significant amount of marine protein, and ${ }^{14} \mathrm{C}$ dates that overlap with those on human remains from other parts of western Scotland that exhibit more terrestrial diets (Richards and Sheridan 2000; Schulting and Richards 2002). These data could be interpreted to indicate that the Cnoc Coig individuals were Mesolithic hunter-gatherers who coexisted with agricultural communities. This highlights the fact that there is insufficient archaeological or other evidence to support a rapid and total abandonment of marine resources in the Early Neolithic, a point emphasized by Milner et al. (2004).

Several stable isotope and lipid biomarker studies have been used to argue for a rapid and permanent shift in Scotland from a diet rich in marine protein in the Mesolithic, to one wholly reliant on terrestrial resources in the Neolithic (Richards et al. 2003; Richards and Schulting 2006; Cramp et al. 2014). However, issues with using stable isotope analyses to detect potentially much more subtle changes in diet have been highlighted by Milner et al. (2004), Bonsall et al. (2009), and Charlton et al. (2016); namely that the use of linear interpolation between terrestrial and marine $\delta^{13} \mathrm{C}$ end-members to estimate marine resource consumption in humans has a large error of up to $20 \%$, and that faunal baselines must be better defined when dealing with humans that may have consumed marine resources. The present study seeks to address both issues.

Our knowledge of the transition is somewhat hindered by the fact that $\delta^{13} \mathrm{C}$ and $\delta^{15} \mathrm{~N}$ isotope analysis of bulk bone collagen is the most cost effective method of estimating individual diets, but the resolution is poor as it gives an average of food sources consumed over 5-10 years or more before the subject's death, depending on which bone is sampled (Sealy et al. 1995; Richards and Hedges 1999). In comparison, the incremental dentine method (Beaumont et al. 2013), which was employed by Montgomery et al. (2013) to detect periodic returns to marine resources by Scottish Neolithic individuals, picks up relatively short-lived changes in eating habits, but only while the teeth are forming. Alternative approaches to reconstructing diet have been utilized in response to the issues associated with analyzing human bone collagen. For example, Cramp et al. (2014) used lipid biomarkers to detect dairy products in Neolithic pot sherds from the Outer Hebrides. In the absence of marine biomarkers, they concluded that dairying completely replaced fishing in the Neolithic. However, the technique can only inform on food cooked in pots (as opposed to on an open fire, for example) and cannot recreate the diet of individual people. Currently, the bulk bone collagen isotopic evidence of diet in the Neolithic in Scotland appears at odds with the archaeological evidence. $\delta^{13} \mathrm{C}$ and $\delta^{15} \mathrm{~N}$ isotope measurements on human bone collagen have been interpreted as indicating an almost complete abandonment of marine resources (Richards et al. 2003; Richards and Schulting 2006), yet Neolithic middens contain evidence of shellfish and fish consumption (Connock et al. 1991). We must therefore seek to reconcile the isotopic and archaeological evidence.

The study of the transition from a nomadic hunter-gatherer-fisher lifestyle to sedentary farming in Scotland is greatly hampered by the scarcity of human and animal remains that survive in the archaeological record. Some dietary resources, such as shellfish are well represented, but the edible organic portion has either been consumed or totally degraded, leaving only the inorganic shells. Organic remains are vital for reconstructing ancient diets and subsistence practices, and for placing these in a secure chronological context. Issues surrounding human diet and lifestyle are particularly pressing in Scotland due to the coastal setting of many of the surviving sites: it is important that these particularly vulnerable sites are recorded and interpreted before they are lost to climate change and erosion. 
The scarcity of suitable bone samples, resulting in unrepresentative dietary baselines in stable isotope studies, is not a problem that is unique to Scotland. Very few stable isotope studies take into account all available food sources when reconstructing diet because of the limited availability of samples to measure. Baselines are constructed from the faunal remains found on site but do not consider foods that may have been lost to degradation. Other studies use nonlocal faunal remains in an attempt to fill baseline gaps, but overlook that one of the fundamental reasons for building a baseline is to correct for natural geographical variations in isotope values that are reflected in every stage of the food-chain (Peterson and Fry 1987). In addition, very small quantities of marine resources in a diet dominated by terrestrial resources are difficult to discern using the traditional stable isotope bivariate plot (e.g. Bonsall et al. 2009). These plots can give a broad indication of dietary habits by examining human isotope values in relation to a faunal baseline. However, relative contributions from different dietary sources cannot be calculated, and no meaningful statistical error can be placed upon dietary estimations. Linear interpolation, using $\delta^{13} \mathrm{C}$ measurements and theoretical $100 \%$ marine and terrestrial human diet $\delta^{13} \mathrm{C}$ end-members, has been utilized as a tool to calculate the abundance of marine protein in the diet (Arneborg et al. 1999; Cook et al. 2015). However, these end-points are commonly derived from populations that may not be relevant to the study and may not be representative of human diets in geographically diverse locations. To detect subtle variations in past diets using bulk bone collagen isotope analysis, we must use geographically relevant samples and interpret the data using models that provide more accurate dietary estimates.

This work used Food Reconstruction Using Isotopically Transferred Signals (FRUITS), a Bayesian mixing model developed by Fernandes et al. (2014) as a tool for reconstructing past human diets. The model works by comparing a mixed target to several isotopically unique sources and calculating the most probable relative contribution each source has made to form the target. The model can be can be used with or without added Bayesian priors. To produce accurate human dietary reconstructions using FRUITS, it is necessary to specify the dietary isotopic baseline values as "food values." These are specified as the values of the food that was consumed, as opposed to the traditionally used faunal bone collagen values, because of the isotopic offset between flesh and bone (Peterson and Fry 1987). However, little consensus exists as to what the correct offsets are and whether they vary between species. The baseline values are specified because natural geological isotope values can vary, and these variations are reflected throughout the food chain from soil/ocean minerals to plants, to herbivores, to carnivores and omnivores. To obtain the correct baseline, it is important to measure the stable isotope values of fauna from the same context as the humans whose diet is being reconstructed. This gives the most accurate baseline values for FRUITS and, indeed, any other method of stable isotope dietary reconstruction.

Carding Mill Bay is a Neolithic shell midden on the west coast of Scotland (Figure 1). The midden contained a variety of faunal and some human remains within a matrix of marine shells dominated by limpets (Patella spp.). Radiocarbon dating places the midden in the period between 4000 and 3500 cal. BC (Connock et al. 1991; Bronk Ramsey et al. 2000). The midden was excavated by Lorn Archaeological Society and finds analysis was carried out by Historic Environment Scotland. Finds included bones of birds, terrestrial mammals (wild herbivores and pig) and marine fish, together with a range of lithic and bone artifacts (Connock et al. 1991). Disarticulated human remains were recovered from several contexts of the midden (IV, VII, X, XIV, XV, and XXIII) and from a cist burial in contexts II and III (Connock et al. 1991). The site has been the subject of several later studies investigating the Scottish Neolithic because of the presence of both human and animal remains as well as "Obanian" artifacts, which are 


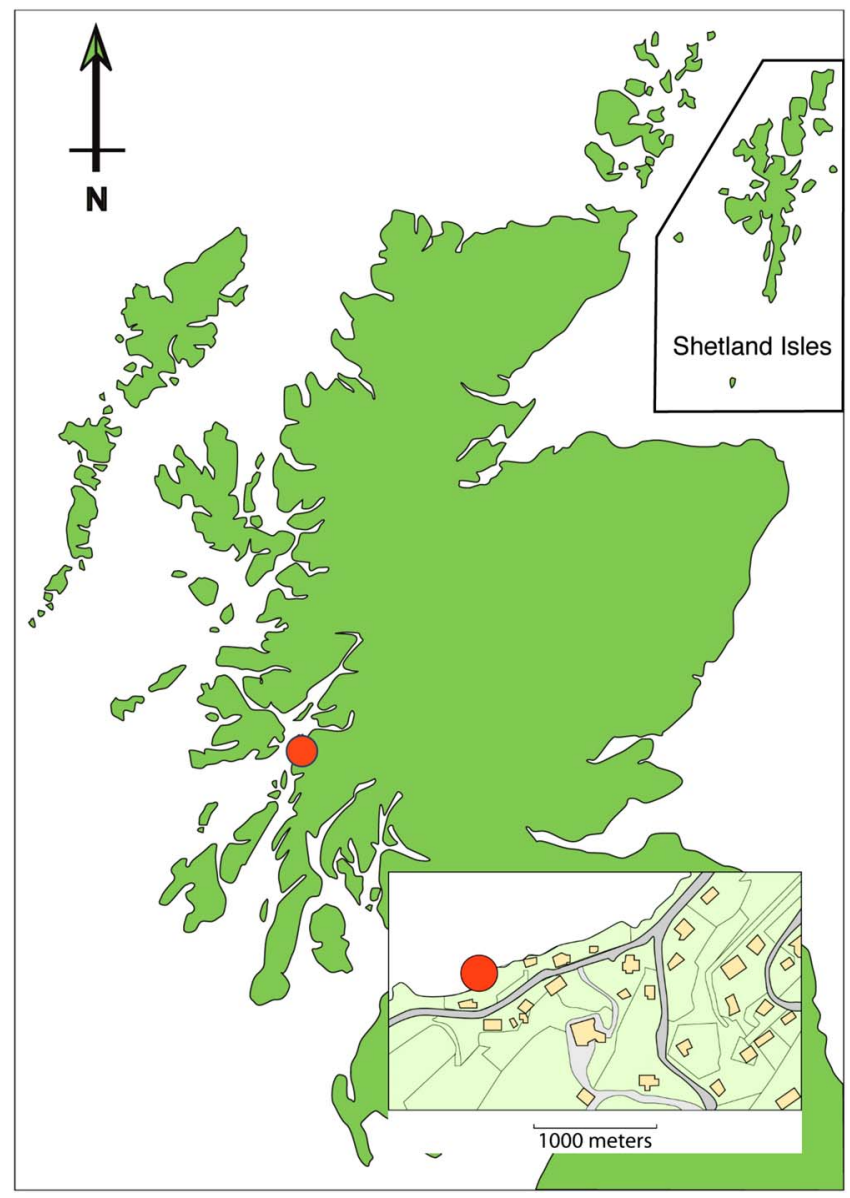

Figure 1 Location of Carding Mill Bay in Scotland.

characteristic bevel ended antler or bone tools, often found in the absence of retouched stone tools (Schulting and Richards 2002; Bartosiewicz et al. 2010).

The work we present here sought to better understand the dietary changes that occurred during the Mesolithic-Neolithic transition in western Scotland, using $\delta^{13} \mathrm{C}, \delta^{15} \mathrm{~N}$, and $\delta^{34} \mathrm{~S}$ isotope measurements on bone collagen from human and animal remains from Carding Mill Bay. Given the sparse character of the faunal assemblage from this site, we tested the hypothesis that modern marine and terrestrial analogs could be used to supplement the ancient archaeological dietary baseline. To ensure the correct values were used in the FRUITS models, we used a local baseline, and measured species-specific $\Delta{ }^{13} \mathrm{C}_{\text {muscle protein-bone collagen }}$ and $\Delta^{15} \mathrm{~N}_{\text {muscle protein-bone collagen offset values }}$ in three marine and four terrestrial species. These values were used to model the diet of four human individuals from the midden whose isotope measurements were previously interpreted to be indicative of a wholly terrestrial diet (Schulting and Richards 2002).

It is well known that the calibration of samples containing marine carbon must take account of the marine reservoir effect (MRE) and that $\Delta \mathrm{R}$ values (representing the local offset from the global average surface water MRE at a point in time) in Scotland show spatial variability 
(Ascough et al. 2004; Cook et al. 2015). Given the uncertainty surrounding marine consumption in the early Scottish Neolithic, a further reason for estimating the marine contribution at Carding Mill Bay was to reassess published ${ }^{14} \mathrm{C}$ dates at the site to take these MREs into account and report more accurate dates for the human samples. This necessitates a calculation of the Carding Mill Bay ${ }^{14} \mathrm{C}$ reservoir offset value $(\Delta \mathrm{R})$ and the proportion of marine carbon contributing to the human bone collagen.

To place the site and the human remains in a more secure chronological context, two contexts were chosen for the determination of new $\Delta \mathrm{R}$ values. The multiple paired marineterrestrial sample approach was adopted (Ascough et al. 2009; Russell et al. 2015) together with $\chi^{2}$ testing (cf. Ward and Wilson 1978) to ensure that the groups of marine and terrestrial samples were internally, statistically indistinguishable. The two contexts chosen for analysis were XIV and XV. Carding Mill Bay has a range of quoted $\Delta \mathrm{R}$ values (see Table 11), however, the values calculated by Reimer et al. (2002) were based on single pairs of samples and since the terrestrial samples employed were charcoal, which can produce anomalously old ages because of the potential for an old wood effect, we made the decision not to use these. The value calculated by Ascough et al. (2004) was identified as an outlier in the complete dataset: this may be because the ${ }^{14} \mathrm{C}$ ages of terrestrial samples fell into two distinct groups and the correct offset was therefore difficult to determine. In light of this, improved values were sought using terrestrial herbivore bone collagen.

\section{METHODS}

Archaeological samples from the midden were provided by Glasgow Museums Research Centre and included 16 samples of at least three terrestrial species including red deer (Cervus elaphus), large mammals [cattle (Bos taurus), and/or red deer (Cervus elaphus)], and medium mammals [sheep (Ovis aries) and/or roe deer (Capreolus capreolus)], to form the isotope baseline for the terrestrial fauna from the site. Contexts with human remains were targeted - see Table 1. As there were no fish bones available to sample from Carding Mill Bay, the ancient marine fish baseline was derived from measurements of Viking Age/Medieval cod from Orkney and Caithness (Russell 2011). Marine fish are highly mobile and this results in very little variation in isotope measurements of fish caught anywhere off the coast of Scotland (Barrett et al. 2008). Therefore, the fish from Orkney were deemed suitable analogs for fish caught at Oban. Modern terrestrial herbivore analogues came from organic farms in Perth and Dumfries (Table 2) Modern marine samples included three species of fish [cod (Gadus morhua), haddock (Melanogrammus aeglefinus) and pollock (Pollachius pollachius)] from the North Sea (Table 3) and four species of shellfish: cockles (Cerastoderma edule), limpets (Patella vulgata), mussels (Mytilus edulis), and winkles (Littorina littorea) from the Oban area (Table 4).

Modern bone samples were split into two fractions: an untreated fraction and a lipid extracted fraction. The lipid-extracted fraction was prepared as follows: each sample was crushed and solvent extracted in a sonic bath for 30 minutes using 2:1 DCM:MeOH (Dichloromethane: Methanol) followed by $\mathrm{MeOH}$ and finally with reverse osmosis water until traces of organic solvent were removed. Collagen was extracted from the lipid-free bone and the bone containing lipids. Ancient faunal bone samples were prepared using the standard SUERC bone collagen pretreatment method with ultrafiltration to remove degraded collagen and other contaminants (Dunbar et al. 2016). Shellfish flesh was Soxhlet extracted for $7 \mathrm{hr}$ using 2:1 DCM:MeOH followed by $7 \mathrm{~h}$ using $\mathrm{MeOH}$. The samples were then thoroughly soaked and washed several times with reverse osmosis water to remove residual organic solvent. Samples for stable isotope 
Table 1 Ancient faunal bone collagen isotope measurements - terrestrial samples from Carding Mill Bay, marine samples from Roberts Haven and Quoygrew (Russell 2011).

\begin{tabular}{|c|c|c|c|c|c|c|c|c|}
\hline Sample ID & Species & $\begin{array}{l}\text { Context } \\
\text { nr }\end{array}$ & $\begin{array}{l}\delta^{13} \mathrm{C}_{\mathrm{VPBD}} \\
(\% \circ)\end{array}$ & $\begin{array}{l}\delta^{15} \mathrm{~N}_{\mathrm{AIR}} \\
(\% 0)\end{array}$ & $\begin{array}{l}\mathrm{C} / \mathrm{N} \\
\text { atomic } \\
\text { ratio }\end{array}$ & $\begin{array}{l}\delta^{34} \mathrm{~S}_{\mathrm{CDT}} \\
(\% \circ)\end{array}$ & $\begin{array}{l}\mathrm{C} / \mathrm{S} \\
\text { atomic } \\
\text { ratio }\end{array}$ & $\begin{array}{l}\mathrm{N} / \mathrm{S} \\
\text { atomic } \\
\text { ratio }\end{array}$ \\
\hline GUsi-3497 & $\begin{array}{l}\text { Medium mammal (Ovis aries or } \\
\text { Capreolus capreolus) }\end{array}$ & VI & -21.6 & +3.5 & 3.2 & +18.7 & 619 & 192 \\
\hline GUsi-3498 & Large mammal (Cervus elaphus or Bos taurus) & IV & -23.3 & +3.4 & 3.2 & +20.8 & 661 & 207 \\
\hline GUsi-3500 & Large mammal (Cervus elaphus or Bos taurus) & IX & -22.8 & +2.8 & 3.5 & +19.6 & 441 & 127 \\
\hline GUsi-3501 & Large mammal (Cervus elaphus or Bos taurus) & IX & -23.1 & +3.1 & 3.5 & N/A & N/A & N/A \\
\hline GUsi-3502 & Large mammal (Cervus elaphus or Bos taurus) & IX & -22.8 & +2.8 & 3.6 & +20.0 & 533 & 149 \\
\hline GUsi-3503 & Large mammal (Cervus elaphus or Bos taurus) & VII & -22.5 & +2.7 & 3.4 & +19.9 & 409 & 121 \\
\hline GUsi-3504 & Large mammal (Cervus elaphus or Bos taurus) & XIV & -22.5 & +3.1 & 3.2 & +20.3 & 580 & 181 \\
\hline GUsi-3505 & Large mammal (Cervus elaphus or Bos taurus) & XIV & -23.2 & +3.7 & 3.3 & N/A & N/A & N/A \\
\hline GUsi-3506 & Large mammal (Cervus elaphus or Bos taurus) & XIV & -22.5 & +2.4 & 3.2 & +21.0 & 699 & 218 \\
\hline GUsi-3507 & $\begin{array}{l}\text { Medium mammal (Ovis aries or } \\
\text { Capreolus capreolus) }\end{array}$ & XIV & -22.9 & +3.7 & 3.2 & +20.1 & 560 & 173 \\
\hline GUsi-3508 & Large mammal (Cervus elaphus or Bos taurus) & XIV & -23.2 & +2.3 & 3.2 & +20.9 & 712 & 223 \\
\hline GUsi-3509 & Red deer (Cervus elaphus) & XVII & -23.2 & +3.0 & 3.4 & +20.3 & 561 & 167 \\
\hline GUsi-3511 & Large mammal (Cervus elaphus or Bos taurus) & XIV & -22.8 & +3.9 & 3.2 & N/A & N/A & N/A \\
\hline GU-18879 & Cod (Gadus morhua) & 3004 & -14.7 & +13.5 & 4.0 & +16.2 & 233 & 58 \\
\hline GU-18880 & Cod (Gadus morhua) & 3004 & -14.1 & +15.3 & 3.8 & +15.9 & 183 & 48 \\
\hline GU-18881 & Cod (Gadus morhua) & 3004 & -13.7 & +13.7 & 3.7 & +16.0 & 199 & 54 \\
\hline GU-18882 & Cod (Gadus morhua) & 3004 & -13.4 & +13.9 & 3.6 & +15.8 & 207 & 57 \\
\hline GU-18883 & Cod (Gadus morhua) & 3019 & -14.4 & +15.0 & 3.6 & +16.5 & 181 & 51 \\
\hline GU-18884 & Cod (Gadus morhua) & 3019 & -12.5 & +13.1 & 3.5 & +15.9 & 206 & 59 \\
\hline GU-18885 & Cod (Gadus morhua) & 3019 & -13.3 & +15.3 & 3.4 & +16.1 & 197 & 57 \\
\hline GU-18886 & Cod (Gadus morhua) & 3019 & -13.3 & +14.0 & 3.4 & N/A & N/A & N/A \\
\hline GU-18887 & Cod (Gadus morhua) & A004 & -12.9 & +13.9 & 3.5 & +16.5 & 210 & 61 \\
\hline GU-18888 & Cod (Gadus morhua) & A004 & -14.3 & +13.7 & 3.6 & +16.9 & 236 & 67 \\
\hline GU-18889 & Cod (Gadus morhua) & A004 & -14.2 & +14.9 & 3.5 & +17.0 & 160 & 44 \\
\hline GU-18890 & Cod (Gadus morhua) & A004 & -13.7 & +13.7 & 3.5 & +16.9 & 282 & 80 \\
\hline GU-18891 & Cod (Gadus morhua) & A023 & -12.4 & +14.4 & 3.3 & +17.1 & 211 & 60 \\
\hline GU-18892 & Cod (Gadus morhua) & A023 & -13.0 & +13.6 & 3.4 & +16.9 & 135 & 41 \\
\hline GU-18893 & Cod (Gadus morhua) & A023 & -13.4 & +13.1 & 3.3 & +16.8 & 226 & 66 \\
\hline GU-18894 & Cod (Gadus morhua) & A023 & -13.0 & +13.8 & 3.4 & +16.8 & 179 & 54 \\
\hline
\end{tabular}


Table 2 Modern inland terrestrial herbivore isotope measurements.

\begin{tabular}{|c|c|c|c|c|}
\hline Species & $\begin{array}{l}\mathrm{Nr} \text { of } \\
\text { samples }\end{array}$ & Tissue fraction & $\begin{array}{l}\text { Average } \\
\delta^{13} C_{\text {VPDB }} \\
\text { value (\%o) }\end{array}$ & $\begin{array}{l}\text { Average } \\
\delta^{15} \mathrm{~N}_{\text {AIR }} \\
\text { value (\%o) }\end{array}$ \\
\hline $\begin{array}{l}\text { Cattle } \\
\text { (Bos taurus) }\end{array}$ & 3 & Bone collagen & $-24.9 \pm 0.2$ & $+6.4 \pm 0.7$ \\
\hline $\begin{array}{l}\text { Cattle } \\
\text { (Bos taurus) }\end{array}$ & 3 & Bone collagen including lipids & $-24.7 \pm 0.0$ & $+6.2 \pm 0.1$ \\
\hline $\begin{array}{l}\text { Cattle } \\
\text { (Bos taurus) }\end{array}$ & 3 & Muscle protein & $-26.6 \pm 0.1$ & $+5.4 \pm 0.6$ \\
\hline $\begin{array}{l}\text { Sheep } \\
\text { (Ovis aries) }\end{array}$ & 6 & Bone collagen & $-25.5 \pm 0.6$ & $+7.3 \pm 0.7$ \\
\hline $\begin{array}{l}\text { Sheep } \\
\text { (Ovis aries) }\end{array}$ & 6 & Bone collagen including lipids & $-25.6 \pm 0.2$ & $+7.6 \pm 0.5$ \\
\hline $\begin{array}{l}\text { Sheep } \\
\text { (Ovis aries) }\end{array}$ & 6 & Muscle protein & $-27.8 \pm 0.1$ & $+7.7 \pm 0.5$ \\
\hline $\begin{array}{l}\text { Red deer } \\
\text { (Cervus elaphus) }\end{array}$ & 10 & Bone collagen & $-24.5 \pm 0.3$ & $+3.4 \pm 0.8$ \\
\hline $\begin{array}{l}\text { Red deer } \\
\text { (Cervus elaphus) }\end{array}$ & 10 & Bone collagen including lipids & $-24.4 \pm 0.3$ & $+3.6 \pm 0.7$ \\
\hline $\begin{array}{l}\text { Red deer } \\
\text { (Cervus elaphus) }\end{array}$ & 10 & Muscle protein & $-26.1 \pm 0.3$ & $+3.7 \pm 0.6$ \\
\hline $\begin{array}{l}\text { Roe deer } \\
\text { (Capreolus capreolus) }\end{array}$ & 9 & Bone collagen & $-24.9 \pm 0.6$ & $+4.9 \pm 1.1$ \\
\hline $\begin{array}{l}\text { Roe deer } \\
\text { (Capreolus capreolus) }\end{array}$ & 9 & Bone collagen including lipids & $-24.7 \pm 1.0$ & $+4.8 \pm 1.4$ \\
\hline $\begin{array}{l}\text { Roe deer } \\
\text { (Capreolus capreolus) }\end{array}$ & 9 & Muscle protein & $-27.1 \pm 0.5$ & $+5.5 \pm 1.3$ \\
\hline
\end{tabular}

analysis were freeze-dried and measured using a Costech ECS 4010 elemental analyzer coupled via a Thermo Scientific Conflo IV to a Thermo Scientific Delta V Advantage continuous-flow isotope ratio mass spectrometer. For $\delta^{13} \mathrm{C}$ and $\delta^{15} \mathrm{~N}$ measurements, approximately $600 \mu \mathrm{g}$ of solid samples were measured alongside gelatine and tryptophan standards. For $\delta^{34} \mathrm{~S}$ measurements, $10 \mathrm{mg}$ of solid samples were measured alongside cysteine, sulphanilamide, and methionine standards.

For determination of the MRE, eight terrestrial herbivore bone collagen samples (four from each of the two contexts) were combusted in sealed quartz tubes and $3 \mathrm{~mL}$ samples of the $\mathrm{CO}_{2}$ were purified cryogenically, graphitized via zinc and iron reduction (Slota et al. 1987) and ${ }^{14} \mathrm{C}$ dated by AMS measurement on the SUERC National Electrostatics Corporation 5MV tandem AMS instrument. For limpet shell samples (again four from each of the two contexts), surface contaminants were removed by first manually cleaning in water and then in a sonic bath within a beaker of water. They were then reacted with $1 \mathrm{M} \mathrm{HCl}$ to remove the outer $20 \%$. The shells were dried, ground, and $0.1 \mathrm{~g}$ subsamples placed in hydrolysis units, where a further $20 \%$ was removed using $1 \mathrm{M} \mathrm{HCl}$ immediately before full hydrolysis of the remaining material. Finally, the shell was hydrolyzed, the $\mathrm{CO}_{2}$ extracted under vacuum and cryogenically purified. $3 \mathrm{~mL}$ subsamples of $\mathrm{CO}_{2}$ were graphitized via zinc and iron reduction before AMS measurement as described above. 
Table 3 Modern local marine fish isotope measurements.

\begin{tabular}{|c|c|c|c|c|}
\hline Species & $\begin{array}{l}\mathrm{Nr} \text { of } \\
\text { samples }\end{array}$ & Tissue fraction & $\begin{array}{l}\text { Average } \\
\delta^{13} C_{\text {VPDB }} \\
\text { value (\%o) }\end{array}$ & $\begin{array}{l}\text { Average } \\
\delta^{15} \mathrm{~N}_{\text {AIR }} \\
\text { value (\%o) }\end{array}$ \\
\hline $\begin{array}{l}\text { Haddock } \\
\text { (Melanogrammus aeglefinus) }\end{array}$ & 10 & Bone collagen & $-14.6 \pm 0.2$ & $+12.4 \pm 0.6$ \\
\hline $\begin{array}{l}\text { Haddock } \\
\text { (Melanogrammus aeglefinus) }\end{array}$ & 10 & $\begin{array}{l}\text { Bone collagen } \\
\text { including lipids }\end{array}$ & $-14.5 \pm 0.4$ & $+12.4 \pm 0.6$ \\
\hline $\begin{array}{l}\text { Haddock } \\
\text { (Melanogrammus aeglefinus) }\end{array}$ & 10 & Muscle protein & $-17.3 \pm 0.2$ & $+12.7 \pm 0.8$ \\
\hline $\begin{array}{l}\text { Cod } \\
\text { (Gadus morhua) }\end{array}$ & 10 & Bone collagen & $-15.1 \pm 0.8$ & $+13.4 \pm 0.5$ \\
\hline $\begin{array}{l}\text { Cod } \\
\text { (Gadus morhua) }\end{array}$ & 10 & $\begin{array}{l}\text { Bone collagen } \\
\text { including lipids }\end{array}$ & $-17.4 \pm 0.8$ & $+13.4 \pm 0.5$ \\
\hline $\begin{array}{l}\text { Cod } \\
\text { (Gadus morhua) }\end{array}$ & 10 & Muscle protein & $-17.8 \pm 0.3$ & $+13.9 \pm 0.3$ \\
\hline $\begin{array}{l}\text { Pollock } \\
\text { (Pollachius pollachius) }\end{array}$ & 10 & Bone collagen & $-15.9 \pm 0.8$ & $+9.6 \pm 0.4$ \\
\hline $\begin{array}{l}\text { Pollock } \\
\text { (Pollachius pollachius) }\end{array}$ & 10 & $\begin{array}{l}\text { Bone collagen } \\
\text { including lipids }\end{array}$ & $-16.6 \pm 0.8$ & $+9.5 \pm 0.3$ \\
\hline $\begin{array}{l}\text { Pollock } \\
\text { (Pollachius pollachius) }\end{array}$ & 10 & Muscle protein & $-18.6 \pm 0.7$ & $+12.7 \pm 0.8$ \\
\hline
\end{tabular}

Table 4 Modern local marine shellfish isotope measurements.

\begin{tabular}{|c|c|c|c|c|}
\hline Species & $\begin{array}{l}\mathrm{Nr} \text { of } \\
\text { samples }\end{array}$ & Tissue fraction & $\begin{array}{l}\text { Average } \\
\delta^{13} C_{\text {VPDB }} \\
\text { value (\%o) }\end{array}$ & $\begin{array}{l}\text { Average } \\
\delta^{15} \mathrm{~N}_{\mathrm{AIR}} \\
\text { value (\%o) }\end{array}$ \\
\hline $\begin{array}{l}\text { Limpet } \\
\text { (Patella vulgata) }\end{array}$ & 13 & Muscle protein & $-15.6 \pm 0.5$ & $+7.6 \pm 1.0$ \\
\hline $\begin{array}{l}\text { Limpet } \\
\text { (Patella vulgata) }\end{array}$ & 13 & Whole muscle & $-16.6 \pm 0.7$ & $+6.9 \pm 0.8$ \\
\hline $\begin{array}{l}\text { Blue mussel } \\
\text { (Mytilus edulis) }\end{array}$ & 8 & Muscle protein & $-16.9 \pm 0.3$ & $+9.2 \pm 0.2$ \\
\hline $\begin{array}{l}\text { Blue mussel } \\
\text { (Mytilus edulis) }\end{array}$ & 8 & Whole muscle & $-18.4 \pm 0.6$ & $+8.7 \pm 0.2$ \\
\hline $\begin{array}{l}\text { Cockle } \\
\text { (Cerastoderma edule) }\end{array}$ & 11 & Muscle protein & $-17.1 \pm 0.5$ & $+9.6 \pm 0.4$ \\
\hline $\begin{array}{l}\text { Cockle } \\
\text { (Cerastoderma edule) }\end{array}$ & 11 & Whole muscle & $-17.8 \pm 0.5$ & $+9.4 \pm 0.5$ \\
\hline $\begin{array}{l}\text { Winkle } \\
\text { (Littorina littorea) }\end{array}$ & 16 & Muscle protein & $-15.3 \pm 0.6$ & $+10.0 \pm 1.2$ \\
\hline $\begin{array}{l}\text { Winkle } \\
\text { (Littorina littorea) }\end{array}$ & 16 & Whole muscle & $-16.0 \pm 0.6$ & $+9.7 \pm 1.4$ \\
\hline
\end{tabular}

Outliers in the age data were initially excluded using a $\chi^{2}$ test; an overall $\Delta \mathrm{R}$ was then calculated using the multiple paired marine/terrestrial sample approach, whereby a $\Delta \mathrm{R}$ value was calculated for all possible pairs and then a weighted mean paired offset was calculated for the context, with 
Table 5 Faunal carbon isotope tissue offset values. Mean values $\pm 1 \sigma$.

\begin{tabular}{|c|c|c|c|c|}
\hline Species & $\begin{array}{l}\text { Nr sample } \\
\text { pairs }\end{array}$ & $\begin{array}{l}\Delta{ }^{13} \mathrm{C}_{\text {muscle protein-bone }} \\
\text { collagen + lipids }\end{array}$ & $\begin{array}{l}\Delta^{13} \mathrm{C}_{\text {muscle protein- }} \\
\text { bone collagen }\end{array}$ & $\begin{array}{l}\Delta^{13} \mathrm{C}_{\text {bone collagen- }} \\
\text { collagen + lipids }\end{array}$ \\
\hline $\begin{array}{l}\text { Cattle } \\
\text { (Bos Taurus) }\end{array}$ & 3 & $-2.0 \pm 0.1$ & $-1.7 \pm 0.1$ & $-0.3 \pm 0.2$ \\
\hline $\begin{array}{l}\text { Sheep } \\
\text { (Ovis aries) }\end{array}$ & 6 & $-2.2 \pm 0.2$ & $-2.2 \pm 0.6$ & $-0.1 \pm 0.6$ \\
\hline $\begin{array}{l}\text { Red deer } \\
\text { (Cervus elaphus) }\end{array}$ & 10 & $-1.7 \pm 0.3$ & $-1.6 \pm 0.4$ & $-0.1 \pm 0.4$ \\
\hline $\begin{array}{l}\text { Roe deer } \\
\text { (Capreolus } \\
\text { capreolus) }\end{array}$ & 9 & $-2.4 \pm 0.8$ & $-2.3 \pm 0.2$ & $-0.2 \pm 0.7$ \\
\hline TERRESTRIAL & 28 & $-2.1 \pm 0.6$ & $-1.9 \pm 0.5$ & $-0.2 \pm 0.5$ \\
\hline $\begin{array}{l}\text { Cod } \\
\text { (Gadus morhua) }\end{array}$ & 10 & $-0.4 \pm 0.8$ & $-2.6 \pm 0.5$ & $+2.2 \pm 0.7$ \\
\hline $\begin{array}{l}\text { Pollock } \\
\text { (Pollachius } \\
\text { pollachius) }\end{array}$ & 10 & $-2.0 \pm 0.2$ & $-2.7 \pm 0.4$ & $+0.7 \pm 0.3$ \\
\hline $\begin{array}{l}\text { Haddock } \\
\text { (Melanogrammus } \\
\text { aeglefinus) }\end{array}$ & 10 & $-2.8 \pm 0.3$ & $-2.7 \pm 0.3$ & $-0.1 \pm 0.4$ \\
\hline MARINE & 30 & $-1.7 \pm 1.1$ & $-2.7 \pm 0.4$ & $+0.9 \pm 1.1$ \\
\hline
\end{tabular}

the variability expressed as the standard error for predicted values (Cook et al. 2015). The human dietary calculations and the new $\Delta \mathrm{R}$ values were then to be used to recalibrate the existing ${ }^{14} \mathrm{C}$ measurements of the human samples using $\mathrm{OxCal} 4.2$, to assess whether any marine consumption detected would have a significant effect on the ages of the human bone collagen.

Published stable isotope values for four human individuals from Carding Mill Bay (Schulting and Richards 2002) were modeled in FRUITS along with the new baseline measurements to create dietary reconstructions. Food values for FRUITS models were calculated using species

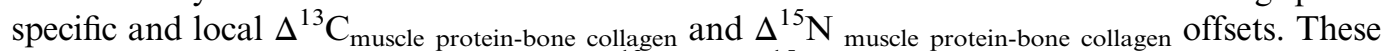
offsets were derived by measuring the $\delta^{13} \mathrm{C}$ and $\delta^{15} \mathrm{~N}$ values of paired flesh-bone collagen samples from representative modern marine and terrestrial species and calculating the difference between flesh and bone values. The mean values of these offsets were used to convert the faunal bone collagen baseline values to food values (see Tables 5 and 6).

\section{RESULTS}

\section{Quality Assurance}

Quality indicators were applied to the isotope measurements after pretreatment and after measurement to ensure that the data were reliable. For ancient bone collagen samples, collagen recovery must be greater than $1 \%$ of the total sample weight and the $\mathrm{C} / \mathrm{N}$ atomic ratio value must be within the range 2.9-3.6 (DeNiro, 1985). Three samples (GU18879, GU18880, and GU18881) were outside of the recommended atomic ratio range and were excluded from further discussion. Quality control indicators for sulphur isotope values for mammal bone collagen are $\mathrm{C}: \mathrm{S}$ atomic ratio $=600 \pm 300$ and $\mathrm{N}: \mathrm{S}$ atomic ratio $=200 \pm 100$, while for fish 
Table 6 Faunal nitrogen isotope tissue offset values. Mean values $\pm 1 \sigma$.

\begin{tabular}{lcccc}
\hline Species & $\begin{array}{l}\text { Nr sample } \\
\text { pairs }\end{array}$ & $\begin{array}{l}\Delta^{15} \mathrm{~N}_{\text {muscle protein- }} \\
\text { bone collagen +lipids }\end{array}$ & $\begin{array}{l}\Delta^{15} \mathrm{~N}_{\text {muscle }} \\
\text { protein-bone collagen }\end{array}$ & $\begin{array}{l}\Delta^{15} \mathrm{~N}_{\text {bone collagen- }} \\
\text { collagen inc. lipids }\end{array}$ \\
\hline $\begin{array}{l}\text { Cattle } \\
\text { (Bos Taurus) }\end{array}$ & 3 & $-0.8 \pm 0.5$ & $-0.9 \pm 0.2$ & $+0.2 \pm 0.7$ \\
$\begin{array}{l}\text { Sheep } \\
\text { (Ovis aries) }\end{array}$ & 6 & $+0.1 \pm 0.9$ & $+0.4 \pm 1.0$ & $-0.3 \pm 0.8$ \\
$\begin{array}{l}\text { Red deer } \\
\text { (Cervus elaphus) }\end{array}$ & 10 & $+0.1 \pm 0.6$ & $+0.3 \pm 0.7$ & $-0.2 \pm 0.2$ \\
$\begin{array}{l}\text { Roe deer } \\
\text { (Capreolus capreolus) }\end{array}$ & 9 & $+0.6 \pm 1.0$ & $+0.5 \pm 0.5$ & $+0.1 \pm 0.8$ \\
$\begin{array}{l}\text { TERRESTRIAL } \\
\text { Cod }\end{array}$ & $\mathbf{2 8}$ & $\mathbf{+ 0 . 2} \pm \mathbf{0 . 9}$ & $\mathbf{+ 0 . 3} \pm \mathbf{0 . 8}$ & $\mathbf{- 0 . 1} \pm \mathbf{0 . 7}$ \\
$\begin{array}{l}\text { (Gadus morhua) } \\
\text { Pollock }\end{array}$ & 10 & $+0.5 \pm 0.3$ & $+0.5 \pm 0.2$ & $-0.0 \pm 0.4$ \\
$\begin{array}{l}\text { Pollachius pollachius) } \\
\text { Haddock }\end{array}$ & 10 & $+0.3 \pm 0.4$ & $+0.2 \pm 0.5$ & $+0.1 \pm 0.2$ \\
$\begin{array}{l}\text { (Melanogrammus } \\
\text { aeglefinus) }\end{array}$ & 10 & $+0.3 \pm 0.4$ & $+0.3 \pm 0.5$ & $-0.0 \pm 0.2$ \\
\begin{tabular}{l} 
MARINE \\
\hline
\end{tabular} & $\mathbf{3 0}$ & $\mathbf{+ 0 . 4} \pm \mathbf{0 . 4}$ & $\mathbf{+ 0 . 4} \pm \mathbf{0 . 4}$ & $\mathbf{- 0 . 0} \pm \mathbf{0 . 2}$ \\
\hline
\end{tabular}

bone collagen they are $\mathrm{C}: \mathrm{S}$ atomic ratio $=175 \pm 50$ and $\mathrm{N}: \mathrm{S}$ atomic ratio $=60 \pm 20$ (Nehlich and Richards 2009). Four samples (GU18879, GU18888, GU18890, GU18893) were outside of these and excluded from further consideration. There are currently no accepted quality indicators for modern flesh or bone collagen including lipid samples measured by CF-IRMS, however we can be confident that the samples were not degraded since they were stored at $-20^{\circ}$ $\mathrm{C}$ before pretreatment and they never came into contact with a burial environment.

\section{Ancient Bone Collagen}

Table 1 illustrates stable carbon, nitrogen and sulphur isotope measurements for herbivore bone collagen from Carding Mill Bay and marine fish from Robert's Haven and Quoygrew. For herbivores, the mean isotope values were: $\delta^{13} \mathrm{C}=-22.8 \pm 0.43 \%, \delta^{15} \mathrm{~N}=+3.1 \pm 0.47 \%$ 。 and $\delta^{34} \mathrm{~S}=+20.0 \pm 0.65 \%$. For marine fish, the values were: $\delta^{13} \mathrm{C}=-13.5 \pm 0.66 \%$ o, $\delta^{15} \mathrm{~N}=$ $+14.1 \pm 0.69 \%$ and $\delta^{34} \mathrm{~S}=+16.5 \pm 0.44 \%$. The $\delta^{13} \mathrm{C}$ and $\delta^{15} \mathrm{~N}$ results are typical of terrestrial herbivores that have been raised on unfertilized land (Bogaard et al. 2013), and wild marine fish (Schoeninger and DeNiro 1984). However, the sulphur isotope measurements made on the herbivores are indicative of a sea-spray effect, whereby marine sulphur is directly ingested by animals that graze near the sea (Wadleigh et al. 1994). For this reason, the sulphur isotope measurements were not used in the FRUITS models to reconstruct human paleodiet.

\section{Modern Terrestrial Isotope Measurements}

Table 2 illustrates average stable carbon and nitrogen isotope measurements for modern terrestrial herbivores that were candidates to supplement the faunal baseline at Carding Mill Bay. These values were used to produce geographically relevant food values for the FRUITS models. The mean isotope values for different tissue fractions were $\delta^{13} \mathrm{C}=$ $-24.9 \pm 0.61 \%$ and $\delta^{15} \mathrm{~N}=+5.2 \pm 1.74 \%$ o for bone collagen, $\delta^{13} \mathrm{C}=-24.8 \pm 0.71 \%$ and 


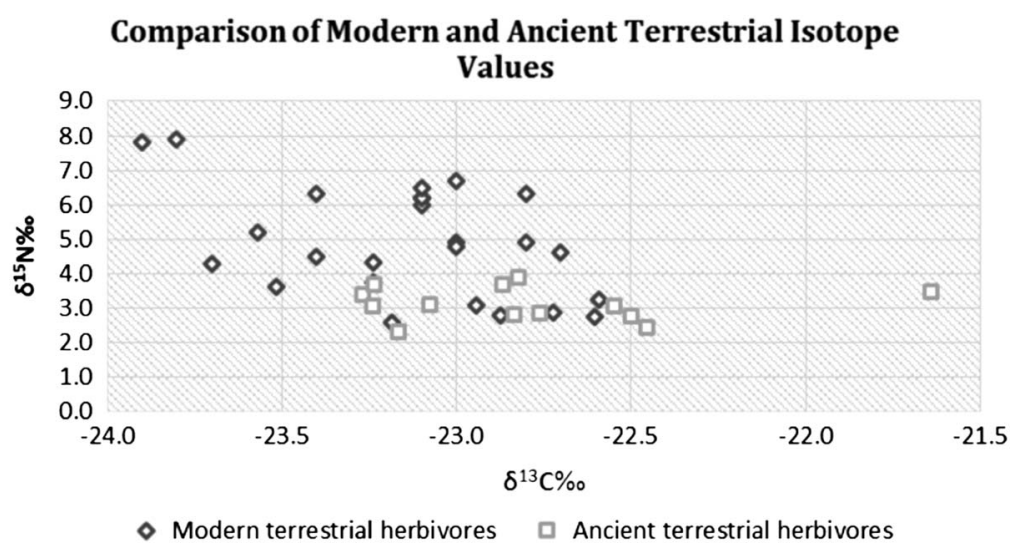

Figure 2 Carbon and nitrogen stable isotope measurements of modern and ancient terrestrial herbivore bone collagen. Modern values corrected for the Suess effect.

$\delta^{15} \mathrm{~N}=+5.2 \pm 1.79 \%$ for bone collagen including lipids, and $\delta^{13} \mathrm{C}=-26.8 \pm 0.71 \%$ and $\delta^{15} \mathrm{~N}=+5.4 \pm 1.70 \%$ for muscle protein.

To account for the atmospheric Suess effect in modern organic samples, whereby organisms are depleted in ${ }^{13} \mathrm{C}$ compared to ancient samples as a result of the release of carbon from fossil fuels in the last two centuries, carbon isotope data must be adjusted by $+1.5 \%$ (Beavan Athfield et al. 2008). Comparing the adjusted modern bone collagen isotope measurements with the measurements from ancient animals at Carding Mill Bay demonstrated that modern and ancient populations are not isotopically comparable (see Figure 2). In addition, there is a large range in the stable nitrogen isotope values of modern herbivore bone collagen $(1 \sigma=1.70)$; this implies large physiological or dietary variations within the modern population that are not present in the ancient population. For this reason, the measurements of modern terrestrial bone collagen were excluded from the FRUITS models as they were deemed incomparable with ancient terrestrial fauna.

\section{Modern Marine Isotope Measurements}

Table 3 summarizes average stable carbon, nitrogen, and sulphur isotope measurements for modern marine fish species, which were candidates to supplement the marine faunal baseline. The mean isotope values for different tissue fractions were: $\delta^{13} \mathrm{C}=-15.2 \pm 0.77 \%$ and $\delta^{15} \mathrm{~N}=$ $+11.8 \pm 1.66 \%$ o for bone collagen, $\delta^{13} \mathrm{C}=-16.1 \pm 1.41 \%$ ond $\delta^{15} \mathrm{~N}=+11.8 \pm 1.72 \%$ or bone collagen including lipids, and $\delta^{13} \mathrm{C}=-17.9 \pm 0.71 \%$ ond $\delta^{15} \mathrm{~N}=+12.1 \pm 1.82 \%$ o for muscle protein.

In contrast to the terrestrial fauna, modern marine fish bone collagen appears to be a good analog for ancient marine bone collagen. Figure 3 shows carbon and nitrogen isotope measurements for modern and ancient cod bone collagen, and modern shellfish protein. The modern data has been corrected to account for the marine Suess effect. There is significant overlap in the isotope data of modern and archaeological cod bone collagen, indicating that chronologically distinct marine populations are isotopically comparable. Since modern and ancient cod bone collagen are comparable, we have made the assumption that modern shellfish flesh samples are also suitable analogs. Isotope data from these samples were used alongside the ancient measurements in FRUITS to build a representative marine dietary isotope baseline. 


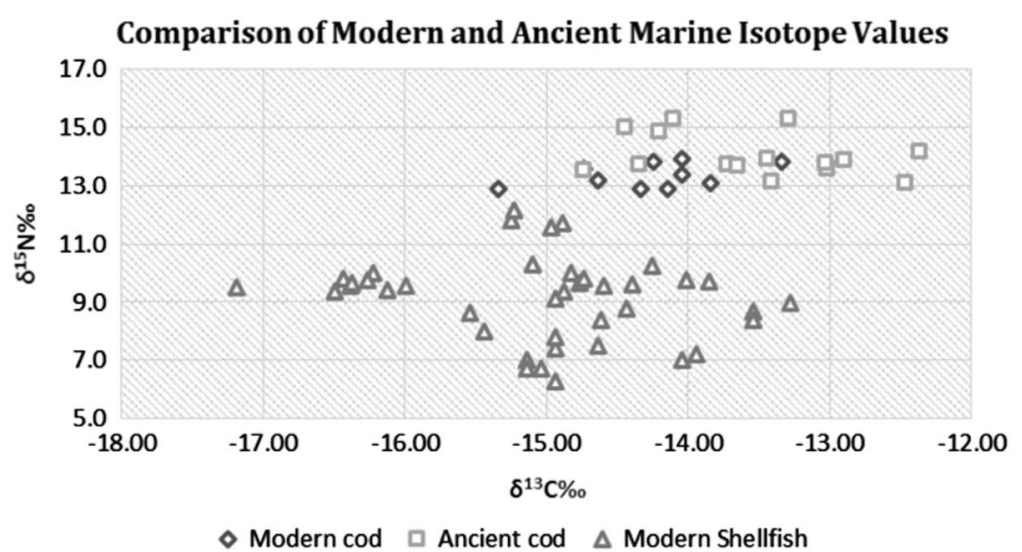

Figure 3 Carbon and nitrogen stable isotope measurements of modern and ancient cod bone collagen, and modern shellfish protein. Modern values corrected for the Suess effect.

\section{Isotopic Tissue Offsets}

While modern terrestrial fauna proved to be unsuitable analogs for ancient samples for the reasons discussed above, stable isotope data from modern animals were useful when calculating tissue offsets that are necessary for modeling diet in FRUITS. Differences in $\delta^{13} \mathrm{C}$ between muscle tissue and bone collagen are related to physiological differences in the tissue structure: lipids are relatively depleted in ${ }^{13} \mathrm{C}$ relative to bone collagen and flesh, and flesh is relatively depleted in ${ }^{13} \mathrm{C}$ compared to bone collagen (Pinnegar and Polunin 1999; Fernandes et al. 2015). These offset values may differ between species, so it is important to use appropriate offsets when reconstructing diet using FRUITS. Isotopic differences between muscle protein and bone collagen in both modern terrestrial samples (Table 2) and modern marine samples (Table 3) were therefore used to produce the $\Delta^{13} \mathrm{C}_{\text {muscle protein-bone collagen }}$ and $\Delta^{15} \mathrm{~N}_{\text {muscle protein-bone collagen }}$ values shown in Tables 5 and 6.

\section{Human Dietary Reconstruction Using FRUITS}

Tables 6, 8, and 9 summarize dietary and ${ }^{14} \mathrm{C}$ data for four human bones (Table 7) recovered from different contexts of the Carding Mill Bay midden. The stable isotope data in Table 8 were used to model dietary intake using FRUITS. To establish whether there was a significant marine reservoir effect in the human bone collagen, the ${ }^{14} \mathrm{C}$ ages given in Table 7 were recalibrated using the marine food source estimates generated by FRUITS and the new $\Delta \mathrm{R}$ value that was obtained (see Tables 8-13).

The modeling parameters considered when reconstructing diet at Carding Mill Bay for each of the human samples were as follows: the protein dietary intake was via the routed model, with carbon and nitrogen isotope values from four consumer bone collagen samples specified with an error of $0.5 \%$ to account for intra-individual variability, following (Fernandes et al. 2015). Tissue offsets applied to obtain the food values were as follows: terrestrial herbivore $\Delta^{13} \mathrm{C}_{\text {muscle protein-bone collagen }}=-1.9$ and $\Delta^{15} \mathrm{~N}_{\text {muscle protein-bone collagen }}=+0.3$, marine fish $\Delta{ }^{13} \mathrm{C}_{\text {muscle protein-bone collagen }}=-2.7$ and $\Delta{ }^{15} \mathrm{~N}_{\text {muscle protein-bone collagen }}=+0.4$. No tissue offsets were required for shellfish muscle protein values used. One dietary fraction (protein) from each of three food groups (terrestrial herbivores, marine fish, and marine shellfish) was then defined 
Table $7{ }^{14} \mathrm{C}$ ages and paleodietary isotope measurements of human bone collagen from Carding Mill Bay (Schulting and Richards 2002).

\begin{tabular}{|c|c|c|c|c|c|}
\hline $\begin{array}{l}\text { Sample } \\
\text { ID }\end{array}$ & $\begin{array}{l}\text { Context } \\
\mathrm{nr}\end{array}$ & $\begin{array}{l}{ }^{14} \mathrm{C} \text { age } \\
(\mathrm{BP})\end{array}$ & $\begin{array}{l}\delta^{13} C_{V P B D} \\
(\% o)\end{array}$ & $\begin{array}{l}\delta^{15} \mathrm{~N}_{\mathrm{AIR}} \\
(\% \circ)\end{array}$ & $\begin{array}{l}\mathrm{C} / \mathrm{N} \\
\text { molar }\end{array}$ \\
\hline OxA-7890 & XXIII & $4330 \pm 60$ & -21.4 & 9.8 & 3.1 \\
\hline OxA-7665 & VII & $4690 \pm 40$ & -21.5 & 9.6 & 3.2 \\
\hline OxA-7664 & XV & $4830 \pm 45$ & -21.0 & 8.9 & 3.1 \\
\hline OxA-7663 & XIV & $4800 \pm 50$ & -21.5 & 9.0 & 3.2 \\
\hline
\end{tabular}

Table $8{ }^{14} \mathrm{C}$ and $\delta^{13} \mathrm{C}$ measurements of marine shell and terrestrial herbivore bone collagen for $\Delta \mathrm{R}$ determination.

\begin{tabular}{llllr}
\hline $\begin{array}{l}\text { Context } \\
\mathrm{nr}\end{array}$ & Sample ID & Sample type & $\begin{array}{l}{ }^{14} \mathrm{C} \text { age } \\
(\mathrm{yr} \mathrm{BP} \pm 1 \sigma)\end{array}$ & $\begin{array}{l}\delta^{13} \mathrm{C} \\
(\% \text { VPDB }\end{array}$ \\
\hline XIV & GU39625 & Terrestrial & $5273 \pm 39$ & -23.3 \\
XIV & GU39626 & Terrestrial & $5190 \pm 36$ & -23.2 \\
XIV & GU39627 & Terrestrial & $5040 \pm 37$ & -23.3 \\
XIV & GU39628 & Terrestrial & $4848 \pm 38$ & -23.4 \\
XIV & GU39633 & Marine & $5413 \pm 28$ & 0.6 \\
XIV & GU39634 & Marine & $5522 \pm 28$ & -0.2 \\
XIV & GU39635 & Marine & $5424 \pm 29$ & 0.2 \\
XIV & GU39636 & Marine & $5395 \pm 28$ & 0.8 \\
XV & GU39629 & Terrestrial & $5334 \pm 39$ & -22.6 \\
XV & GU39630 & Terrestrial & $5320 \pm 39$ & -23.3 \\
XV & GU39631 & Terrestrial & $5155 \pm 39$ & -23.4 \\
XV & GU39632 & Terrestrial & $5278 \pm 36$ & -22.2 \\
XV & GU39637 & Marine & $5519 \pm 28$ & 0.6 \\
XV & GU39638 & Marine & $5467 \pm 29$ & 0.4 \\
XV & GU39639 & Marine & $5575 \pm 29$ & 0.9 \\
XV & GU39640 & Marine & $5573 \pm 28$ & 0.1 \\
\hline
\end{tabular}

Table 9 Results of $\chi^{2}$ tests on all ${ }^{14} \mathrm{C}$ ages for terrestrial and marine samples from contexts XIV and XV.

\begin{tabular}{llr}
\hline Context $\mathrm{nr}$ & Terrestrial $\chi^{2} \mathrm{~T}$ value & Marine $\chi^{2} \mathrm{~T}$ value \\
\hline XIV & $72.10\left(\chi_{: 0.05}^{2}=7.81\right)$ & $12.39\left(\chi_{2}^{2}: 0.05=7.81\right)$ \\
$\mathrm{XV}$ & $13.07\left(\chi^{2}: 0.05=7.81\right)$ & $9.56\left(\chi^{2}: 0.05=7.81\right)$ \\
\hline
\end{tabular}

using the average stable isotope values and standard error of the mean of the modern faunal muscle protein: $\delta^{13} \mathrm{C}_{\text {herbivores }}=-24.7 \pm 0.1 \%$ and $\delta^{15} \mathrm{~N}_{\text {herbivores }}=+3.4 \pm 0.1 \%$ o, $\delta^{13} \mathrm{C}_{\text {fish }}=$ $-16.8 \pm 0.1 \%$ and $\delta^{15} \mathrm{~N}_{\text {fish }}=+12.9 \pm 0.3 \%$, and $\delta^{13} \mathrm{C}_{\text {shellfish }}=-15.2 \pm 0.1 \%$ and $\delta^{15} \mathrm{~N}_{\text {shellfish }}=$ $+9.5 \pm 0.2 \%$. These values have been corrected to account for the marine Suess effect: modern marine carbon isotope data must be adjusted by $0.86 \%$ (Beavan Athfield et al. 2008). Chosen diet to collagen offsets were $1 \pm 1 \%$ ofor $\delta^{13} \mathrm{C}$ and $5.5 \pm 0.5 \%$ ofor $\delta^{15} \mathrm{~N}$. Table 12 and Figure 4 illustrate the results of dietary reconstruction using FRUITS. 
Table 10 Data for contexts XIV and XV that contained inconsistent measurements on the basis of $\chi^{2}$ tests.*

\begin{tabular}{|c|c|c|c|c|c|}
\hline Context nr & $\begin{array}{l}\text { Consistent } \\
\text { measurements }\end{array}$ & $\begin{array}{l}\text { Age } \\
\mathrm{BP} \pm 1 \sigma\end{array}$ & $\begin{array}{l}\text { Inconsistent } \\
\text { measurements }\end{array}$ & $\begin{array}{l}\text { Age } \\
\mathrm{BP} \pm 1 \sigma\end{array}$ & $\begin{array}{l}\mathrm{T} \\
\text { value }\end{array}$ \\
\hline $\begin{array}{l}\text { XIV } \\
\text { (terrestrial) }\end{array}$ & $\begin{array}{l}\text { No consis } \\
\text { measuremen } \\
\text { therefore, a j } \\
\text { made to incluc } \\
\text { when calcula }\end{array}$ & $\begin{array}{l}\text { restrial } \\
\text { identified; } \\
\text { nt call was } \\
\text { easurements } \\
\Delta \text { R value. }\end{array}$ & $\begin{array}{l}\text { GU39625 } \\
\text { GU39626 } \\
\text { GU39627 } \\
\text { GU39628 }\end{array}$ & $\begin{array}{l}5273 \pm 39 \\
5190 \pm 36 \\
5040 \pm 37 \\
4848 \pm 38\end{array}$ & 72.10 \\
\hline $\begin{array}{l}\text { XIV } \\
\text { (marine) }\end{array}$ & $\begin{array}{l}\text { GU39633 } \\
\text { GU39635 } \\
\text { GU39636 }\end{array}$ & $\begin{array}{l}5413 \pm 28 \\
5424 \pm 29 \\
5935 \pm 28\end{array}$ & GU39634 & $5522 \pm 28$ & 3.55 \\
\hline $\begin{array}{l}\text { XV } \\
\text { (terrestrial) }\end{array}$ & $\begin{array}{l}\text { GU39629 } \\
\text { GU39630 } \\
\text { GU39632 }\end{array}$ & $\begin{array}{l}5334 \pm 39 \\
5320 \pm 39 \\
5278 \pm 36\end{array}$ & GU39631 & $5155 \pm 39$ & 1.23 \\
\hline $\begin{array}{l}\text { XV } \\
\text { (marine) }\end{array}$ & $\begin{array}{l}\text { GU39637 } \\
\text { GU39639 } \\
\text { GU39640 }\end{array}$ & $\begin{array}{l}5519 \pm 28 \\
5575 \pm 29 \\
5573 \pm 28\end{array}$ & GU39638 & $5467 \pm 29$ & 2.54 \\
\hline
\end{tabular}

${ }^{*}$ Consistent measurements were used to calculate values of $\Delta \mathrm{R}$. T-statistics shown are for consistent groups.

Table $11 \Delta \mathrm{R}$ values for Carding Mill Bay from literature sources and this study.

\begin{tabular}{lrl}
\hline Context $\mathrm{nr}$ & $\Delta \mathrm{R}$ Value & Source \\
\hline XIII & $+150 \pm 28$ & Ascough et al. (2007) \\
XIV & $-44 \pm 91$ & Reimer et al. (2002) \\
XIV & $-130 \pm 34$ & This research \\
XV & $+86 \pm 67$ & Reimer et al. (2002) \\
\hline
\end{tabular}

Table 12 Summary data for human diet at Carding Mill Bay derived from FRUITS.

\begin{tabular}{llll}
\hline Sample & $\begin{array}{l}\text { Marine fish } \\
\text { contribution (\%) }\end{array}$ & $\begin{array}{l}\text { Marine shellfish } \\
\text { contribution (\%) }\end{array}$ & $\begin{array}{l}\text { Terrestrial herbivore } \\
\text { contribution (\%) }\end{array}$ \\
\hline OxA-7890 & $8 \pm 6$ & $13 \pm 8$ & $79 \pm 7$ \\
OxA-7665 & $7 \pm 5$ & $12 \pm 8$ & $81 \pm 7$ \\
OxA-7664 & $6 \pm 5$ & $10 \pm 7$ & $84 \pm 6$ \\
OxA-7663 & $5 \pm 4$ & $10 \pm 7$ & $85 \pm 6$ \\
\hline
\end{tabular}

\section{Recalibrating Radiocarbon Dates at Carding Mill Bay}

$\Delta \mathrm{R}$ values were calculated for contexts XIV and XV at the site. For context XIV marine samples, one sample (GU39634) was excluded as an outlier and the remaining three samples passed the $\chi^{2}$ test. However, the terrestrial samples failed the $\chi^{2}$ test completely, with no two samples passing. On that basis, we determined that context XIV is mixed and unsuitable for deriving a $\Delta \mathrm{R}$ value. For context XV, one marine and one terrestrial sample (GU39638 and GU39631, respectively) were removed and the remaining three samples in both groups passed 
Table 13 Summary of recalibrated ${ }^{14} \mathrm{C}$ dates of humans from Carding Mill Bay. Reported with $95 \%$ confidence interval.

\begin{tabular}{lll}
\hline Sample & $\begin{array}{l}\text { Previous age } \\
\text { range (cal BC) }\end{array}$ & $\begin{array}{l}\text { Recalibrated age } \\
\text { range (cal BC) }\end{array}$ \\
\hline OxA-7890 & $3096-2878$ & $3089-2680$ \\
OxA-7665 & $3632-3366$ & $3625-3333$ \\
OxA-7664 & $3698-3524$ & $3661-3379$ \\
OxA-7663 & $3660-3384$ & $3646-3376$ \\
\hline
\end{tabular}
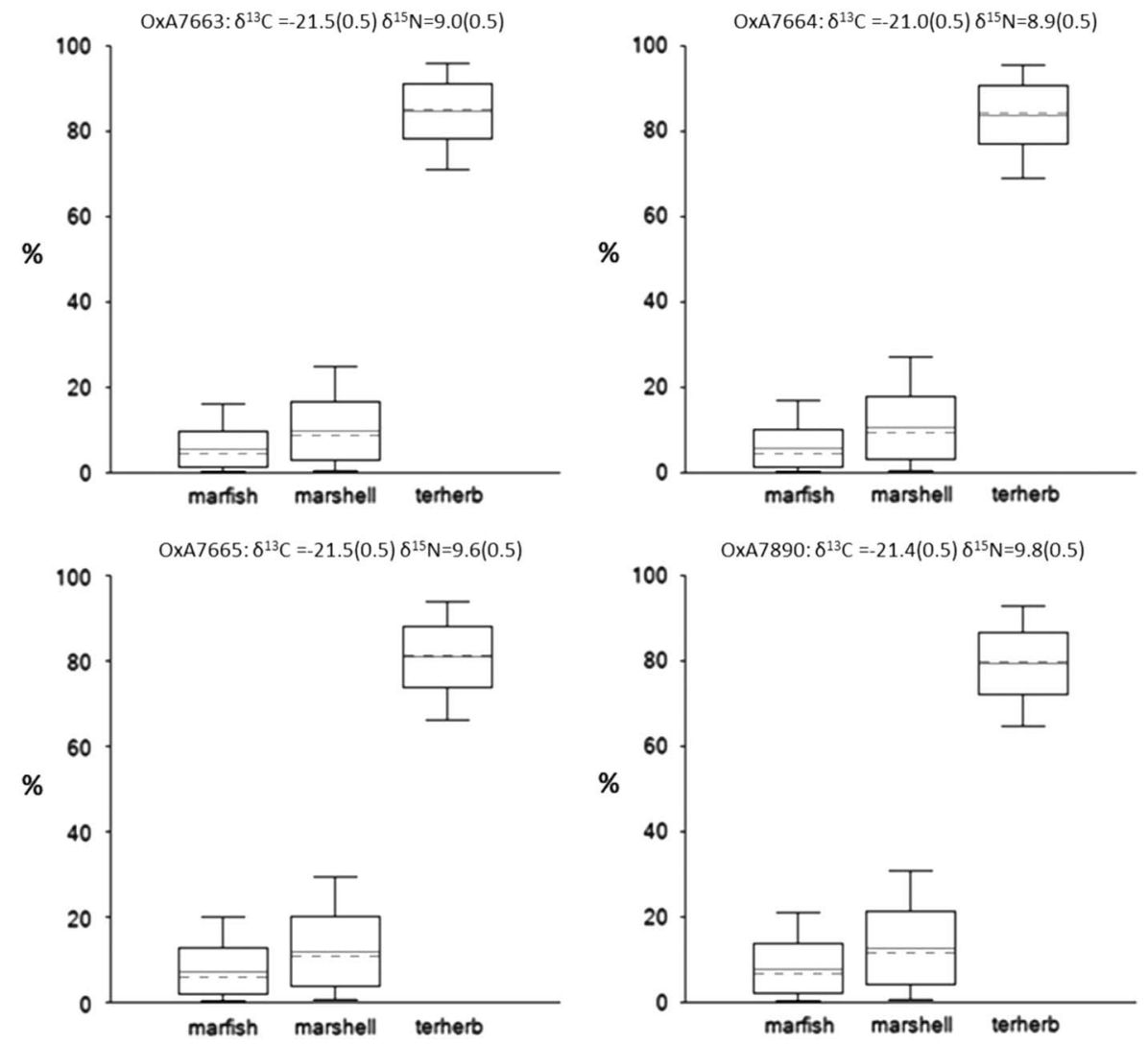

Figure 4 FRUITS reconstructions of human diet at Carding Mill Bay. Boxes represent a $68 \%$ credible interval and whiskers represent a $95 \%$ credible interval. Within the boxes, the solid line represents the estimated median and the dashed line represents the estimated mean.

the $\chi^{2}$ test. In this case, context $\mathrm{XV}$ was deemed suitable for $\Delta \mathrm{R}$ calculation. The calculated value was $-130 \pm 34{ }^{14} \mathrm{C}$ yr.

The ${ }^{14} \mathrm{C}$ ages for the four humans at Carding Mill Bay were first calibrated using the terrestrial calibration curve within OxCal 4.2, to provide baseline ${ }^{14} \mathrm{C}$ dates (Reimer et al. 2013). The ages were then recalibrated using the mixed marine/terrestrial calibration curve within OxCal 4.2 (Reimer et al. 2002), employing the marine dietary information for each individual obtained from FRUITS, together with the recalculated $\Delta \mathrm{R}$ value. See Figure 5 for all ${ }^{14} \mathrm{C}$ calibrations for this site. 

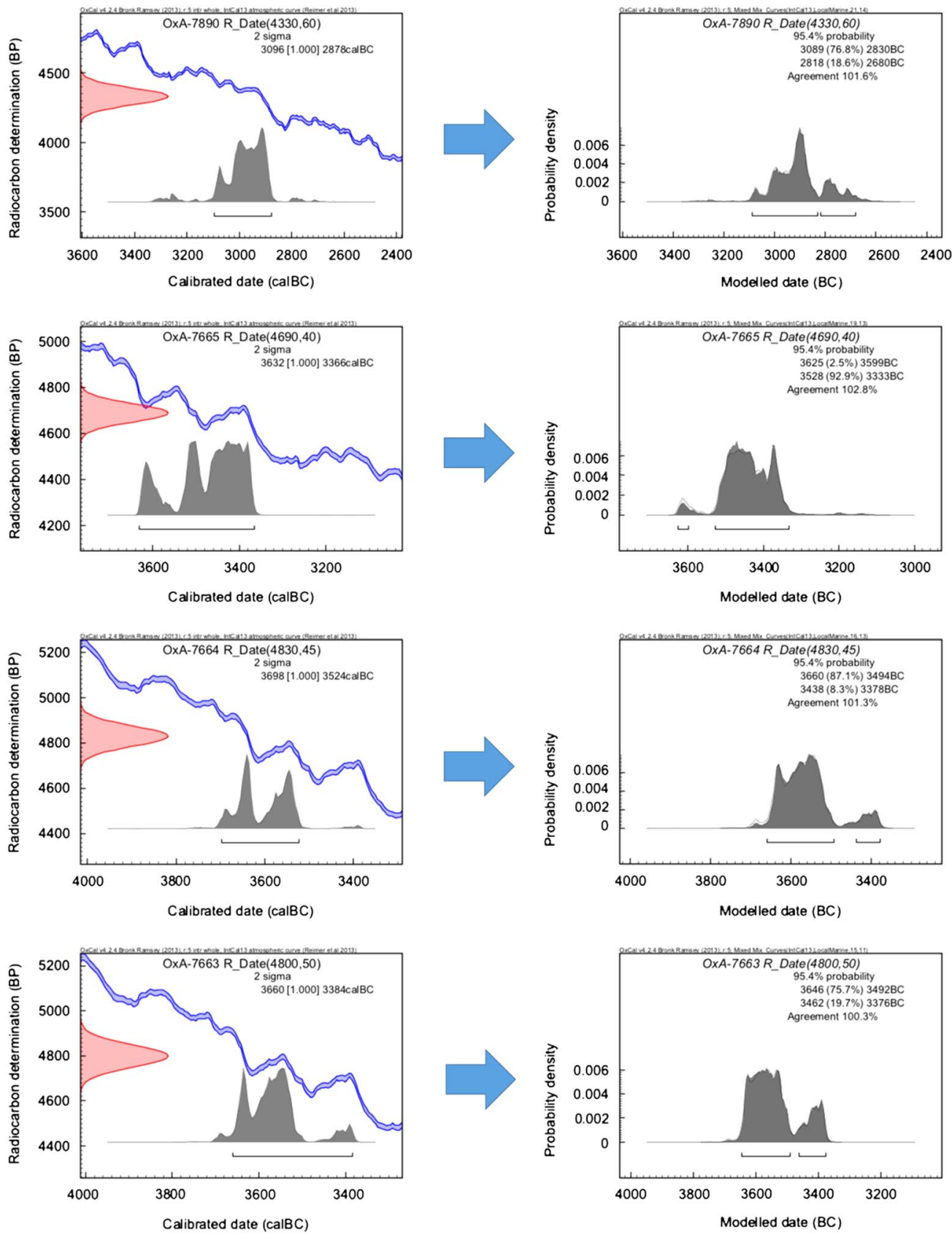

Figure 5 Recalibrated radiocarbon dates of humans from Carding Mill Bay using an updated $\Delta \mathrm{R}$ value of $-130 \pm 34{ }^{14} \mathrm{C}$ yr and percentage marine contribution to diet calculated using FRUITS.

\section{DISCUSSION}

There are three strands of the investigation that require discussion: the dietary habits and dates of the human remains at Carding Mill Bay, the subsistence strategies of the population, and the novel use of FRUITS to model Neolithic diet in western Scotland. 
First, and most significantly, the results of the FRUITS reconstruction of all four humans reveal modest amounts $(15 \pm 11$ to $21 \pm 14 \%$ ) of marine resources in the diet. This is significant, given that the site and the human samples are dated to the Neolithic, when exploitation of marine foods had supposedly been abandoned in favor of farming (Richards et al. 2003). Our results are in contrast to previous interpretations of bulk bone isotope data that suggested a complete absence of marine resources in the Neolithic diet (Richards et al. 2003; Richards and Schulting 2006; Cramp et al. 2014), but are in agreement with the archaeological record for the site.

In all human samples analyzed, we see a diet dominated by terrestrial resources, but supplemented with marine fish and shellfish. The models suggest that shellfish were a more important resource at Carding Mill Bay than marine fish, and this is reflected in the archaeological record, with shellfish being far more abundant than fish remains (Connock et al. 1991). It seems likely that the use of marine resources at this time would have been secondary to farm produce, either as a supplement to the diet or as a famine food in times of crop/livestock failure. The employment of shellfish gathering being prioritized over fishing is sensible: shellfish were easy to source with very little skill, and required no tools more complicated than a rock to dislodge them. Fishing, on the other hand, required specialist skill and equipment, took more time, and quite probably yielded fewer rewards. If Early Neolithic communities exploited wild marine resources, it is sensible that they would choose the simpler and more rewarding over the difficult and time consuming.

The findings at Carding Mill Bay have parallels with results derived from high-resolution measurements of stable isotopes in tooth dentine from Neolithic individuals in Shetland (Montgomery et al. 2013). These showed that at least during their childhood years they periodically and temporarily returned to eating marine resources before reverting back to a more terrestrial-based diet. The authors hypothesized that this was in response to difficulties encountered while attempting to maintain a sedentary farming culture in a marginal environment (Montgomery et al. 2013). While our bulk bone collagen isotope values cannot reveal periodic returns to marine resources at different stages in life, they do show small inputs of marine resources to the diet, which is also evident in the results from Shetland.

Following our recalibration of the four ${ }^{14} \mathrm{C}$ ages derived from human bone collagen, to take into account the improved $\Delta \mathrm{R}$ value for the site and the potential marine reservoir effect caused by the consumption of shellfish and fish, we find very little shift in the ages. While Figure 5 and Table 13 show that the recalibrated ages are less precise, our assertion is that they are more accurate. This is noteworthy, since, even after correction of the ages, these individuals date to the first half of the Scottish Neolithic; not only do we demonstrate that the chronology of the site is reliable and secure, but we show that fish and shellfish in the diet can be firmly associated with the Neolithic.

Further information about subsistence practices at Carding Mill Bay can be derived from the stable isotope data of the Neolithic terrestrial herbivores. The sulphur isotope measurements of all herbivores at the site indicate a marine signal, demonstrating the ingestion of sulphur indirectly from seawater, most commonly described as the "sea-spray effect" (Wadleigh et al. 1994). This effect, caused by animals grazing close to a coastline, as observed here, suggests that the animals were probably raised locally and not moved from inland sites to the Oban area. The low $\delta^{15} \mathrm{~N}$ values for the herbivore bone collagen also suggest that the land on which these animals grazed was not fertilized with manure, and the low $\delta^{13} \mathrm{C}$ values indicate they were not foddered with seaweed (Bogaard et al. 2013). While we can be confident of the latter point 
because significant foddering would result in a slight "marine signature" to the $\delta^{13} \mathrm{C}$ values, we cannot say the same of the former since comparison of nitrogen isotope values from pre-Neolithic herbivores would be required to be sure that the low values are not simply as a result of a natural geographical variation in isotopes.

We can also make some observations about FRUITS as a dietary reconstruction model. The accuracy and reliability of FRUITS has been demonstrated in several past studies (Fernandes et al. 2014, 2015; Sayle et al. 2016), however, the application of modeling parameters has always been at the user's discretion. $\Delta{ }^{13} \mathrm{C}_{\text {muscle protein-bone collagen }}$ and $\Delta^{15} \mathrm{~N}_{\text {muscle protein-bone collagen }}$ offset values used here have been specifically calculated from tissue fractions of animals of the same species as those analyzed in the isotope faunal baseline. Offsets are reasonably comparable across species from the same environment and the error associated with each value indicates that they are relatively accurate representations of tissue offsets (see Tables 4 and 5). The use of empirically derived offsets in FRUITS removes any uncertainty associated with values calculated from unrepresentative species and geographically incomparable sites, thus strengthening the reliability of the models in this study.

Finally, we have demonstrated the ability of FRUITS to distinguish between the consumption of different food groups from the same environment. Traditional interpretations of stable carbon and nitrogen isotope plots are unable to identify the consumption of shellfish separately from the consumption of fish. Indeed, dietary interpretations based on a standard linear mixing model never even consider shellfish as a potential food source since they cannot be included in a bone collagen faunal baseline. The abundance of shellfish remains in coastal middens in Scotland necessitates their consideration as a part of the Neolithic diet. The fact that the isotopic food value of shellfish differs in $\delta^{15} \mathrm{~N}$ enrichment from fish suggests they should be treated as a distinct food source in future dietary studies.

\section{CONCLUSIONS}

At Carding Mill Bay, we demonstrate how the use of stable isotope measurements from human and animal bone collagen, realistic collagen/muscle isotope offsets, and the Bayesian mixing model (FRUITS) for determining paleodiet reveals the presence of small amounts of marine resources in the diet of Early Neolithic humans recovered from this site. Our consideration of archaeological and stable isotope evidence from Neolithic western Scotland suggests that the dietary shifts from marine to terrestrial resources were not as rapid or absolute as has been suggested. Finally, we can make certain suggestions about farming practices at this coastal site, with evidence showing that animals at Carding Mill Bay may have been of local origin and that foddering and fertilization practices, observed in later Neolithic sites, were not present.

Our results have implications both for dietary research using stable isotopes and for archaeological investigations of shell middens. When building a picture of ancient diet using stable isotopes, it is important to consider all available dietary evidence from the site and include this in a dietary baseline. The importance of using dietary baselines is well established, but we must ensure that they are also representative of the diet consumed by the human individuals analyzed. Where there is evidence of dietary habits, but a lack of samples suitable for stable isotope analysis, appropriate modern analogs should be used to increase sample size. We must also consider the appropriate model to use when estimating ancient diets. In sites like Carding Mill Bay, with evidence of varying subsistence practices, it is essential to use a Bayesian mixing model such as FRUITS, which is capable of discerning small contributions of different food groups. 


\section{ACKNOWLEDGMENTS}

The authors thank the staff of the SUERC radiocarbon and AMS laboratories for assistance with sample pretreatment, stable isotope, and ${ }^{14} \mathrm{C}$ measurements. We also thank Ricardo Fernandes for the time spent providing training and advising on the appropriate use of FRUITS. The assistance of Sheena Fraser with archaeozoological analysis of faunal samples is gratefully acknowledged. We thank two anonymous reviewers for their comments on this paper. Samples for analysis were obtained in collaboration with staff of the Glasgow Museums Research Centre and Historic Environment Scotland. Funding for this research was provided by the AHRC (Grant no. AH/L008521/1).

\section{REFERENCES}

Arneborg J, Heinemeier J, Lynnerup N, Nielson HL, Rud N, Sveinbjörnsdóttir ÁE. 1999. Change of diet of the Greenland Vikings determined from stable carbon isotope analysis and ${ }^{14} \mathrm{C}$ dating of their bones. Radiocarbon 41(2):157-68.

Ascough PL, Cook GT, Dugmore AJ, Barber J, Higney E, Scott EM. 2004. Holocene variations in the Scottish marine radiocarbon reservoir effect. Radiocarbon 46(2):611-20.

Ascough PL, Cook GT, Dugmore AJ, Scott EM. 2007. The North Atlantic marine reservoir effect in the early Holocene: implications for defining and understanding MRE values. Nuclear Instruments and Methods in Physics Research B 259:438-47.

Ascough P, Cook GT, Dugmore AJ. 2009. North Atlantic marine ${ }^{14} \mathrm{C}$ reservoir effects: implications for late-Holocene chronological studies. Quarternary Geochronology 4:171-80.

Barrett J, Johnstone C, Harland J, Van Neer W, Ervynck A, Makowiecki D, Heinrich D, Hufthammer AK, Enghoff IB, Amundsen C. 2008. Detecting the medieval cod trade: a new method and first results. Journal of Archaeological Science 35:850-61.

Bartosiewicz L, Zapata L, Bonsall C. 2010. A tale of two shell middens: the natural versus the cultural in "Obanian" deposits at Carding Mill Bay, Oban, western Scotland. In: VanDerwarker A, Peres TM, editors. Integrating Zooarchaeology and Paleoethnobotany. New York: Springer-Verlag.

Beavan Athfield N, Green RC, Craig J, McFadgen B, Bickler S. 2008. Influence of marine sources on ${ }^{14} \mathrm{C}$ ages: isotopic data from Watom Island, Papua New Guinea inhumations and pig teeth in light of new dietary standards. Journal of the Royal Society of New Zealand 38:1-23.

Beaumont J, Gledhill A, Lee-Thorp J, Montgomery J. 2013. Childhood diet: a closer examination of the evidence from dental tissues using stable isotope analysis of incremental human dentine. Archaeometry 55:277-95.

Bogaard A, Fraser R, Heaton TH, Wallace M, Vaiglova P, Charles M, Jones G, Evershed RP, Styring AK, Andersen NH. 2013. Crop manuring and intensive land management by Europe's first farmers. Proceedings of the National Academy of Sciences 110:12589-94.
Bonsall C, Cook G, Pickard C, McSweeney K, Bartosiewicz L. 2009. Dietary trends at the Mesolithic-Neolithic transition in northwest Europe. Chronology and evolution in the Mesolithic of northwest Europe: 539-62.

Bronk Ramsey C. 2009. Bayesian analysis of radiocarbon dates. Radiocarbon 51(1):337-60.

Bronk Ramsey C, Pettitt PB, Hedges REM, Hodgins GWL, Owen DC. 2000. Radiocarbon dates from the Oxford AMS system: Archaeometry Datelist 30. Archaeometry 42:459-79.

Charlton S, Alexander M, Collins M, Milner N, Mellars P, O'Connell TC, Stevens RE, Craig OE. 2016. Finding Britain's last hunter-gatherers: a new biomolecular approach to 'unidentifiable' bone fragments utilising bone collagen. Journal of Archaeological Science 73:55-61.

Connock KD, Finlayson B, Mills CM. 1991. Excavation of a shell midden site at Carding Mill Bay near Oban, Scotland. Glasgow Archaeological Journal 17:25-38.

Cook GT, Ascough PL, Bonsall C, Hamilton WD, Russell N, Sayle KL, Scott EM, Bownes JM. 2015. Best practice methodology for ${ }^{14} \mathrm{C}$ calibration of marine and mixed terrestrial/ marine samples. Quaternary Geochronology 27:164-71.

Cramp LJE, Jones J, Sheridan A, Smyth J, Whelton H, Mulville J, Sharples N, Evershed RP. 2014. Immediate replacement of fishing with dairying by the earliest farmers of the northeast Atlantic archipelagos. Proceedings of the Royal Society B 281: 20132372.

DeNiro MJ. 1985. Post mortem preservation and alteration of in vivo bone collagen isotope ratios in relation to palaeodietary reconstruction. Nature 317:806-9.

Dunbar E, Cook G, Naysmith P, Tripney B, Xu S. 2016. AMS ${ }^{14} \mathrm{C}$ Dating at the Scottish Universities Environmental Research Centre (SUERC) Radiocarbon Dating Laboratory. Radiocarbon. $1-15$.

Fernandes R, Grootes P, Nadeau MJ, Nehlich O. 2015. Quantitative diet reconstruction of a Neolithic population using a Bayesian mixing model (FRUITS): the case study of Ostorf (Germany). American Journal of Physical Anthropology 158:325-40. 
Fernandes R, Millard AR, Brabec M, Nadeau M-J, Grootes P. 2014. Food reconstruction using isotopic transferred signals (FRUITS): a Bayesian model for diet reconstruction. PloS one 9:e87436.

Haak W, Balanovsky O, Sanchez JJ, Koshel S, Zaporozhchenko V, Adler CJ, Der Sarkissian CS, Brandt G, Schwarz C, Nicklisch N. 2010. Ancient DNA from European early neolithic farmers reveals their near eastern affinities. PLoS Biol 8:e1000536.

Milner N, Craig OE, Bailey GN, Pedersen K, Andersen SH. 2004. Something fishy in the Neolithic? A re-evaluation of stable isotope analysis of Mesolithic and Neolithic coastal populations. Antiquity 78:9-22.

Montgomery J, Beaumont J, Jay M, Keefe K, Gledhill AR, Cook GT, Dockrill SJ, Melton ND. 2013. Strategic and sporadic marine consumption at the onset of the Neolithic: increasing temporal resolution in the isotope evidence. Antiquity 87:1060-72.

Nehlich O, Richards MP. 2009. Establishing collagen quality criteria for sulphur isotope analysis of archaeological bone. Archaeological and Anthropological Sciences 1:59-75.

Olalde I, Schroeder H, Sandoval-Velasco M, Vinner L, Lobón I, Ramirez O, Civit S, Borja PG, SalazarGarcía DC, Talamo S. 2015. A common genetic origin for early farmers from Mediterranean Cardial and Central European LBK cultures. Molecular biology and evolution 32:3132-42.

Peterson BJ, Fry B. 1987. Stable isotopes in ecosystem studies. Annual Review of Ecology and Systematics 18:293-320.

Pinnegar J, Polunin N. 1999. Differential fractionation of $\delta^{13} \mathrm{C}$ and $\delta^{15} \mathrm{~N}$ among fish tissues: implications for the study of trophic interactions. Functional Ecology 13:225-31.

Reimer P, McCormac F, Moore J, McCormick F, Murray E. 2002. Marine radiocarbon reservoir corrections for the midto late Holocene in the eastern subpolar North Atlantic. The Holocene 12:129-35.

Reimer PJ, Bard E, Bayliss A, Beck JW, Blackwell PG, Bronk Ramsey C, Buck CE, Cheng H, Edwards RL, Friedrich M. 2013. IntCall3 and Marine13 radiocarbon age calibration curves 0-50,000 years cal BP. Radiocarbon 55(4):1869-87.

Richards MP, Hedges RE. 1999. Stable isotope evidence for similarities in the types of marine foods used by Late Mesolithic humans at sites along the Atlantic coast of Europe. Journal of Archaeological Science 26:717-22.

Richards M, Mellars P. 1998. Stable isotopes and the seasonality of the Oronsay middens. Antiquity $72: 178-84$.
Richards MP, Schulting RJ. 2006. Touch not the fish: the Mesolithic-Neolithic change of diet and its significance. Antiquity 80:444-56.

Richards MP, Schulting RJ, Hedges RE. 2003. Archaeology: sharp shift in diet at onset of Neolithic. Nature 425:366.

Russell N. 2011. Marine radiocarbon reservoir effects (MRE) in archaeology: temporal and spatial changes through the Holocene within the UK coastal environment. University of Glasgow.

Russell N, Cook GT, Ascough P, Scott EM. 2015. A period of calm in Scottish seas: A comprehensive study of $\Delta R$ values for the northern British Isles coast and the consequent implications for archaeology and oceanography. Quaternary Geochronology 30:34-41.

Sayle KL, Hamilton WD, Gestsdóttir H, Cook GT. 2016. Modelling Lake Mývatn's freshwater reservoir effect: utilisation of the statistical program FRUITS to assist in the re-interpretation of radiocarbon dates from a cemetery at Hofstaðir, north-east Iceland. Quaternary Geochronology 36:1-11.

Schoeninger MJ, Deniro MJ. 1984. Nitrogen and carbon isotopic composition of bone collagen from marine and terrestrial animals. Geochimica et Cosmochimica Acta 48:625-39.

Schulting RJ, Richards MP. 2002. The wet, the wild and the domesticated: the Mesolithic-Neolithic transition on the west coast of Scotland. European Journal of Archaeology 5:147-89.

Sealy J, Armstrong R, Schrire C. 1995. Beyond lifetime averages: tracing life histories through isotopic analysis of different calcified tissues from archaeological human skeletons. Antiquity 69: 290-300.

Sheridan A. 2010. The Neolithization of Britain and Ireland: The 'Big Picture'. In: Finlayson B, Warren G, editors. Landscapes in Transition. Oxford: Oxbow Books.

Slota P, Jull AT, Linick T, Toolin L. 1987. Preparation of small samples for ${ }^{14} \mathrm{C}$ accelerator targets by catalytic reduction of CO. Radiocarbon 29:303-6.

Thomas J. 2008. The mesolithic-neolithic transition in Britain. In: Pollard J, editor. Prehistoric Britain. Oxford: Blackwell.

Wadleigh M, Schwarcz H, Kramer J. 1994. Sulphur isotope tests of seasalt correction factors in precipitation: Nova Scotia, Canada. Water, Air, and Soil Pollution 77:1-16.

Ward GK, Wilson SR. 1978. Procedures for comparing and combining radiocarbon age determinations: a critique. Archaeometry 20:19-31. 